\title{
MANUAL FOR AUTHORS OF MATHEMATICAL PAPERS ${ }^{1}$
}

1. Introduction. Our purpose is to advise mathematicians about preparing papers for publication so as to improve the readability and appearance of the printed article, and to eliminate unnecessary delay, trouble, and expense in the printing.

The crucial difficulty is that an uninformed author frequently makes severe demands on the skill of the compositor to achieve trivial effects. Notations convenient for handwriting are often troublesome and costly when printed (e.g. a tilde over several symbols); and some of the advantages of printing are used too little (e.g. boldface). This is a serious problem. The cost of setting a page of mathematics in type varies from $\$ 10$ to $\$ 30$ depending on the difficulties posed by the manuscript. The difference between an informed, careful author and his opposite can average $\$ 15$ per page. An editor is very conscious of this problem. In a marginal case, his judgment of a paper may be swayed by this non-mathematical feature.

The information in this manual should enable authors to adapt to the medium of print, and to make only intelligent use of the compositor's skill. The most important section is headed: Selecting notations.

2. Organizing a paper (for beginners). The usual journal article is aimed at experts and near-experts, who are the people most likely to read it. Your viewpoint should be to say quickly what you have done that is good, and why it works. Avoid lengthy summaries of known results, and minimize the preliminaries to the statements of your main results. There are many good ways of organizing a paper which can be learned by studying papers of the better expositors. The following suggestions describe a standard acceptable style.

Choose a title which helps the reader place the paper in the body of mathematics. A useless title: Concerning some applications of a theorem of $J$. Doe. A good title contains several well-known key words, e.g. Algebraic solutions of linear partial differential equations. Make the title as informative as possible; but avoid redundancy, and eschew the medieval practice of letting the title serve as an inflated advertisement. A title of more than ten or twelve words is likely to be miscopied, misquoted, distorted, and cursed.

1 This manual was prepared by a committee whose members were Professors J. L. Doob, Leonard Carlitz, F. A. Ficken and George Piranian, with Professor Norman E. Steenrod as chairman. 
The first paragraph of the introduction should be comprehensible to any mathematician, and it should pinpoint the location of the subject matter. The main purpose of the introduction is to present a rough statement of the principal results. This should be done as soon as feasible, although it is sometimes well to set the stage with a preliminary paragraph. The remainder of the introduction can discuss the connections with other results.

It is sometimes useful to follow the introduction with a brief section that establishes notation and refers to standard sources for basic concepts and results. Normally this section should be less than a page in length. Some authors weave this information unobtrusively into their introductions, avoiding thereby a dull section.

The next section should contain the statement of one or more principal results. The rule that the statement of a theorem should precede its proof applies equally well to the main results. It is usually poor practice to postpone the statement of a main result until a series of partial results makes its proof a triviality. A reader wants to know the objective toward which he is heading, and the relevance of each section as he reads it. In the case of a major theorem whose proof is long, its statement can be followed by an outline of the proof with references to subsequent sections for proofs of the various parts.

Strive for proofs that are conceptual rather than computational. For an example of the difference, see A Mathematician's Miscellany, by J. E. Littlewood*, in which the contrast between barbaric and civilized proofs is beautifully and amusingly portrayed. To achieve conceptual proofs, it is of ten helpful for the author to adopt initially the attitude he would take if he could only communicate his mathematics orally (as when walking with a friend). Decide how to state your results with a minimum of symbols, and how to express the ideas of the proof without computations. Then add to this framework the details needed to clinch the results.

Omit any computation which is routine (i.e. does not depend on unexpected tricks). Merely indicate the starting point, describe the procedure, and state the outcome.

It is good research practice to analyze an argument by breaking it into a succession of lemmas, each stated with maximum generality. It is usually bad practice to try to publish such an analysis, since it is likely to be long and uninteresting. The reader wants to see the path-not examine it with a microscope. A part of the argument is worth isolating as a lemma if it is used at least twice later on.

The rudiments of grammar are important. The few lines that you write on the blackboard during an hour's lecture are augmented by spoken commentary, and at the end of the day they are washed away

* Published by Methuen Co. Ltd., London, 1953; see page 30. 
by a merciful janitor. Since your published paper will forever speak for you, without benefit of the cleansing sponge, careful attention to sentence structure is worthwhile. Each author must develop a style that suits him; a few general suggestions are nevertheless appropriate.

The barbarism called the dangling participle has recently become more prevalent, but not less loathsome. "Differentiating both sides with respect to $x$, the equation becomes ..." is wrong, because "the equation" cannot be the subject that does the differentiation. Write instead "Differentiating both sides with respect to $x$, we get the equation ...., or "Differentiation of both sides with respect to $x$ leads to the equation ...."

Although the notion has gained some currency, it is absurd to claim that the informal "we" has no proper place in mathematical exposition. Strict formality is appropriate in the statement of a theorem, and casual chatting should indeed be banished from those parts of a paper which will be printed in italics. But fifteen consecutive pages of formality are altogether foreign to the spirit of the twentieth century, and nearly all authors who try to sustain an impersonal dignified text of such length succeed merely in erecting elaborate monuments to clumsiness.

A sentence of the form "if $P, Q$ " can be understood. However "if $P, Q, R, S, T$ " is not so good, even if it can be deduced from the context that the third comma is the one that serves the role of "then." The reader is looking at your paper to inform himself, and not with a desire for mental calisthenics.

3. Typesetting. Most mathematics is set on monotype machines. The compositor works at a keyboard. The pressing of a key results in the casting from a matrix of a single symbol in type which is then placed on a line, next to its predecessor. If the entire job can be done by pressing keys, the happy state of minimum cost is achieved.

Unfortunately the monotype has various limitations. When a place is reached where the machine is inadequate, the compositor inserts a blank and goes on. Subsequently, the blank must be located, removed, and replaced by a hand-cast symbol. The pressing of a key costs a fraction of a penny. Handwork is much more expensive.

One limitation is that the compositor cannot backspace, as on a typewriter, and place one symbol above another on the same line. Thus fractions cause difficulty, also subscripts under superscripts, and any inflections over subscripts.

Another limitation is that the monotype cannot use the matrix of a symbol whose height exceeds that of the standard line, e.g. oversize integral and summation signs, and also large parentheses, braces, and brackets.

The most important limitation is that the machine has only 255 
keys. Compare this with the fact that there are about 1000 different symbols which an article in mathematics may use. Prior to composition, each manuscript is examined to determine which 255 symbols are used most frequently; and the matrices for these are placed in the monotype. All other symbols must then be hand-set for each occurrence.

4. Selecting notations. There are numerous ways in which the limitations described above can be avoided without sacrifice of clarity, and in most cases with an increase of clarity. The first is to strive for conceptual proofs as opposed to computational ones (see \$2). This will prevent an unnecessary proliferation of special symbols, and it surely makes for easier reading.

Obtain a list of the symbols available on the typewriter to be used in typing the manuscript, and select notations from this list as much as possible. This has the added advantage of minimizing the filling in by hand of blanks in the typed copy.

It is not necessary to represent symbolically each concept which appears. If a concept occurs rarely, it is usually better to refer to it by name. For example, the expression "the upper derivative on the right of $f$ at $a$ " compared with

$$
\limsup _{x \rightarrow a+} \frac{f(x)-f(a)}{x-a}
$$

is just as clear, takes less space, and is very much less trouble to set in type. The purpose of a symbolism is to provide ready reference to objects so as to reveal the structure of an argument in a clear and compact fashion. If the symbols do not contribute to this purpose, use words instead.

It is a common tendency to use distinct symbols for distinct objects throughout the same paper. This is sometimes not necessary. If in $\$ 5$ it is stated that $\alpha$ represents a complex number, this cancels all previous usages. Consistency of notation is necessary only for the important concepts recurring throughout the paper.

Subsubscripts, sub-to-superscripts, etc., are called indices of the second order. They should be used sparingly and with care. In the first place, they are more difficult to read, especially by those who need stronger glasses. The typist of the manuscript is frequently uncertain where to put them; and the compositor can't be more certain. How is he to know that a typewritten $x^{b} h$ means $x^{b h}$ ? Also, there are certain combinations which, when properly printed, do not give the intended effect. For example, $A$-sub- $\gamma$-sub- $b$ looks like this: $A_{\gamma_{b}}$; and $A$-super- $b$-super- $\gamma$ looks like $A^{b^{\gamma}}$. At first glance one can be misled 
until it is recalled that $b$ and $\gamma$ on the same level have the appearance $b \gamma$. Third order indices are not available.

Any large-scale consistent use of second-order indices should be avoided, and it usually can be, to the advantage of all concerned. For example, the monstrosity

$$
\sum_{i_{1}, i_{2}, \cdots, i_{k}=1}^{n} a_{i_{1} i_{2} \cdots i_{k}} \Phi^{i_{1} i_{2} \cdots i_{k}}
$$

can be replaced by

$$
\sum_{\alpha \in S(n, k)} a_{\alpha} \Phi^{\alpha}
$$

where $S(n, k)$ denotes the set of sequences of length $k$ from the integers $1, \cdots, n$. It is even preferable to write

$$
\sum a_{\alpha} \Phi^{\alpha} \quad(\alpha \in S(n, k)) .
$$

In the period 1900-1930, differential geometers developed and popularized an elaborate notation in which most of the information is communicated through systematic use of indices. The virtue of the notation is that it reduces many calculations to routine inspection and control of indices. Its disadvantages are many. It is expensive to print, hard to see, and prone to typographical errors. Also, it tends to emphasize technical details of computation at the expense of conceptual notions. Some of the more recent writers in the subject have successfully avoided the worst features of the old notation. It would be well if others would study their efforts, and follow suit.

Table I presents a list of alternatives to expensive notations. Any large-scale use of the expensive notations should be avoided. In most cases the trouble is caused by placing one symbol above another. In any displayed formula where much handwork is needed for other reasons, a bit of additional handwork is a minor matter. Thus the lefthand notations of Examples 6 through 10 are acceptable in a line which must be handset, but should be avoided in text or in displayed lines not otherwise requiring handsetting.

Example 5 is an instance of the general rule: Write $e^{u}$ if $u$ is simple, but write $\exp (u)$ if $u$ is complicated.

In connection with Example $6, \sqrt{ } 3$ is preferable to $3^{1 / 2}$.

Most printers have special matrices for Latin letters with bars or tildes. Thus if one barred letter, say $\bar{C}$, occurs frequently enough, its matrix can be included among the 255 used in the monotype. In contrast, if each barred letter of the alphabet were used just once, then all would be handset. 
Bars, tildes, and carets on subscripts or superscripts must be handset. Composite matrices are not available. These should therefore be avoided, or used very rarely.

A bar which covers two or more letters must always be handset. If this occurs in the text, then the space between lines must be increased to accommodate the bar. A few of these would be of no concern; but, in case of a great many, the closure symbol Cl, as in Example 12 , is recommended. A tilde or caret covering two or more symbols is too costly to be considered.

Boldface characters can be called for by underscoring the typed letter with a black wiggly line (or tilde). However, boldface is not usually available in subscripts and superscripts.

\section{TABLE I}

Expensive notations

1. $\bar{A}, \tilde{b}, \check{\gamma}, \hat{g}, \stackrel{\AA}{\Lambda}$

2. $\vec{v}$

3. $\varlimsup$ lim, $\underline{\lim }$

4. $\stackrel{\lim , \lim }{\longleftarrow}$

5. $e^{-\frac{x^{2}+y^{2}}{a^{2}}}$

6. $\sqrt{a^{2}-b^{2}}$

7. $\frac{7}{8}, \frac{a+b}{c}$

8. $\sum_{i=0}^{n}, \prod_{i=1}^{\infty}$

9. $\overline{A \cap B}$

10. $A \stackrel{f}{\rightarrow} B$

Alternatives
$A^{\prime}, b^{\prime \prime}, \gamma^{*}, g_{*}, \Lambda^{\#}$
v (boldface)

lim sup, lim inf

inj lim, proj lim

$\exp \left(-\left(x^{2}+y^{2}\right) / a^{2}\right)$

$\sqrt{ }\left(a^{2}+b^{2}\right)$ or $\left(a^{2}+b^{2}\right)^{1 / 2}$

$7 / 8,(a+b) / c$

$\sum_{i=0}^{n}, \quad \prod_{i=1}^{\infty}$

$\mathrm{Cl}(A \cap B)(\mathrm{Cl}=$ closure $)$

$f: A \rightarrow B$

11. $\frac{\cos \frac{1}{x}}{\sqrt{a+\frac{b}{x}}}$

12. $\bar{f}^{-1}$

$$
\frac{\cos (1 / x)}{(a+b / x)^{1 / 2}}
$$

13. $e_{i_{1} i_{2}} \cdots i_{n}$

$f^{-1}$

14. $d_{\breve{a}}, d_{\bar{c}}$

$e\left(i_{1}, i_{2}, \cdots, i_{n}\right)$

$d_{a^{\prime}}, d_{e^{\prime \prime}}$ 
5. Displays, diagrams, figures, and tables. A short and simple formula should be left in the line of text and not displayed unless it must be numbered for later reference. A formula whose length is nearly half a line should be displayed; because, otherwise, it is likely to be broken with part on one line and part on the next, and may thus be difficult to decipher. Compositors are requested, when breaking a formula, to try to do so immediately after an equality sign or one of the other relation symbols. Display any formula which is $1 \frac{1}{2}$ inches long or more and has no convenient breaking point. Authors are urged to avoid long paragraphs with many formulas but no displays. The reason for this is explained in the second paragraph of $\$ 10$.

Some authors number every displayed line of their typescript. The practice is unfortunate for two reasons: If all displayed lines are numbered, the numbers lose their value as clear signals and signposts (they are reduced to the status of section markers); and if the printer finds it necessary to display a few of the formulas that the author had left in the text, the few exceptions stand out as singularities and spoil the author's meticulous routine.

Diagrams of groups and homomorphisms in which the homomorphisms are represented by arrows can be set by the compositor if they are properly arranged. The compositor can readily build a long vertical or horizontal line with an arrowhead at one end; and with considerable difficulty he can piece together a $45^{\circ}$ line. But he cannot construct lines at other angles or make curved lines. Since a diagram can usually be arranged in many ways, the author should choose the one which is simplest to set in type. If the diagram cannot be arranged simply, then it must be treated as an illustration or figure.

Illustrations and figures should be carefully drawn in black ink (india ink) on strong white paper so that photo-engravings can be made from them (blue ink does not photograph successfully). It is customary to reduce the figure to half its size in the process; and the author should take this into account in making the drawing-especially the lettering.

In matrices and tables, vertical alignment is important; and it is essential that the manuscript exhibit the desired alignment clearly. A poorly-typed manuscript can easily mislead the compositor.

It is expensive and wasteful to enclose matrices in long curving lines (oversize parentheses). It is recommended that the matrix be enclosed by a pair of vertical lines, and that a determinant be indicated by prefixing "det"; thus $\left|a_{i j}\right|$ denotes the matrix, and det $\left|a_{i j}\right|$ its determinant. A second good method is to enclose the matrix in brackets $\left[a_{i j}\right]$, and to use $\left|a_{i j}\right|$ for its determinant. A third acceptable method uses $\left\|a_{i j}\right\|$ for the matrix, and $\left|a_{i j}\right|$ for the determinant. 
There is a serious difficulty that may arise with any matrix, diagram, figure, table or displayed formula which occupies more than four lines. If the author specifies its exact location in the text, then the compositor, while cutting the galley into pages, may find it necessary to cut it in two parts with the first part at the bottom of one page and the second at the top of the next. This will destroy vertical alignment, and may render the material almost unreadable. To avoid this difficulty, the author should label the diagram as a figure, and refer to it as such in the text. This allows the compositor to move it to a nearby place to avoid breaking it.

6. Bibliography. Items in the bibliography are usually ordered alphabetically by name of author, and they are numbered consecutively. The names of journals should be abbreviated. Standard abbreviations for some of the principal American journals are

Amer. J. Math.

Amer. Math. Monthly

Ann. of Math.

Bull. Amer. Math. Soc.

Canad. J. Math.

Comm. Pure Appl. Math.

Duke Math. J.

Illinois J. Math.
J. Math. Mech.

J. Symbolic Logic

Math. Reviews

Michigan Math. J.

Pacific J. Math.

Proc. Amer. Math. Soc.

Proc. Nat. Acad. Sci. U.S.A.

Trans. Amer. Math. Soc.

A full list of standard abbreviations can be found in the annual index of Math. Reviews. The name of the journal is followed in order by the volume number, the year, and the first and last page numbers, thus:

3. J. Doe, Summability of Fourier series, Pacific J. Math. 12 (1960), 232-257.

Observe that the month of issue and the issue number are not included. Some journals have discontinued an older numbering of volumes and started afresh with volume one, adding a serial number to distinguish the new block of volumes. In such cases, the serial number should be listed in parentheses before the volume number: Ann. of Math. (2) 52 (1950), 127-139.

A reference to a book should give in order author, title, edition (if not the first), name of series and number (if one of a series), publisher (or distributor), city, and year:

4. F. Hausdorff, Mengenlehre, 3rd ed., Göschens Lehrbuch. 7, W. de Gruyter, Berlin, 1935. 
A reference in the text to the bibliography is customarily of the form: "It is well known [5, pp. 32-34] that . . .," or "According to a result of J. Doe [3, Th. 7, p. 252], ..." Authors are urged to make references specific by including page numbers. The extra space and trouble is fully justified by the saving to the readers.

7. Footnotes. These are more expensive than equivalent material embodied in the text because, being in smaller type, all footnotes are set together in a separate operation. Subsequently, during the paging, they must be separated and inserted in their proper locations. Because of the smaller type, complicated formulas in footnotes must be avoided.

It is therefore best to have no footnotes. The usual literary excuse for a footnote (to avoid interrupting the continuity) does not apply here because the reading of mathematics is usually painfully discontinuous. It is increasingly the custom (and editors and their staffs applaud this warmly) to incorporate a footnote into the text, perhaps parenthetically, perhaps as a "Remark." Acknowledgements can be given in a paragraph at the end of the introduction.

If footnotes are deemed necessary, then all footnotes should be typed together on a single page at the end of the manuscript, numbered consecutively. The reference number in the text should be signalled by writing e.g. "footnote 2 " in the margin. An initial footnote beginning with "Presented to..." or "Work done under contract ..." need not be numbered.

8. The typing of the manuscript. It is important that the manuscript be typed with at least double spacing, and with generous margins on both sides ( $1 \frac{1}{4}$ inches). The double spacing provides clear separation of subscripts and superscripts, and also permits the author to insert changes directly above portions crossed out. The margins allow the technical editor to write instructions to the printer.

The pages of the manuscript should be numbered consecutively in the upper right corners. This insures that the absence of a misplaced page will be noticed.

The manuscript that goes to the printer must be of good quality paper. It is subjected to handling of sufficient roughness to cause thin paper to tear. A carbon copy is very unsatisfactory for two reasons: The paper is usually too thin, and moisture from the hand causes the letters to smear.

It is increasingly the custom to submit manuscripts produced by mimeographing or some other duplicating process. This is advantageous to an author, for it permits him to circulate his paper to a 
sizable audience a year before publication. Such a manuscript is acceptable for printing if it satisfies the above requirements: at least double spacing, generous margins, good quality paper, clear letters, and non-smear ink. A well-executed mimeographed or multilithed manuscript is satisfactory. Other methods are almost always unsatisfactory.

The time for deciding on the precise arrangement of the displayed formulas comes just before the typing. Sloppiness in the handwritten formulas tends to perpetuate itself throughout the typed and printed versions because no one but the author is competent to make alterations. Compositors and typists can only endeavor to reproduce the copy before them. Thus, for each display, the author should visualize exactly what he wants in the printed version, and then he should produce a reasonable facsimile which exhibits clearly the alignment and spacing of all its parts, both horizontally and vertically.

It is very helpful if the typed manuscript follows the standard format of journal articles in the matter of indentations of section headings and theorems. If the handwritten copy does not do this, then the typist should be asked to follow standard practice as exemplified by a reprint.

9. Preparing the manuscript. Because typewriters do not have enough symbols, it is necessary for authors to fill in quite a few formulas by hand. This must be done carefully, in ink, so that the compositor will know what is wanted. The first requirement is that the typist leave enough space, horizontally and vertically, for the handwritten symbols. (Inexperienced typists tend to leave too little space.) Secondly, the author should give extra thought and care to the placing of the symbols. The compositor does not understand mathematics, and he is guided solely by what he sees in the manuscript. He works along lines, and he must know what is on the line, what is a subscript, a superscript, and a subsubscript. He cannot guess that a hastily scribbled gij means $g_{i j}$. Also, the manuscript should distinguish clearly between similar symbols such as

$$
\cup, \mathrm{u}, \mathrm{U} ; \quad 0,0,0 ; x, \times ; \phi, \varnothing ; l, 1 ; \epsilon, \in \text {. }
$$

It is very helpful if, in any doubtful case, the author explains his wishes by means of a pencilled note in the margin.

Small changes in the typed copy (a few words here and there) can be carefully handwritten in black or blue-black ink in the spaces between lines. Do not write lengthwise along the margin. Do not use proofreading symbols as if the typed copy were galley proof. Larger alterations should be typed. For example, the lower half of page 5 
can be crossed out, and a marginal note can say: Insert here the material on page $5 \mathrm{a}$. In deleting material, cross it out, and do not tear off part of a page. A half page may not stay in the paper clip and may be lost. Do not use scotch tape on the top side of a page, for nobody can write on it.

The author can call for special type by underlining according to the following conventions:

Italic -a straight underline in black,
Boldface-a wavy underline in black,
Greek - a straight underline in red,
German - a straight underline in green,
Script - encircle the letter in blue.

The author should leave to the editorial assistant the underlining of the title, section headings, and the statements of theorems, lemmas, and corollaries. Also, do not underline mathematical symbols which are to appear in the usual italic. The only italic underlining the author should do is that of the occasional word or sentence in the text he may wish to emphasize. Do not underline the special symbols

$$
\sum \text { (sum), } \Pi \text { (product), } \in \text { (is an element of), } \varnothing \text { (empty set). }
$$

German letters and script capitals are obtained most conveniently by typing the letter and marking as indicated above. A second method is to fill in a fair approximation by hand, and then, on a separate page of instructions to the printer, make a table of the handwritten symbols adjacent to the corresponding typed symbols.

The page of instructions to the printer should also list alternatives for any special symbols the printer might not have.

The author should submit a manuscript which he believes to be complete. If errors are discovered after submission, typewritten replacement pages should be sent to the editor as soon as possible. Once a manuscript has been set in type, over half the cost of publication has been expended. If, at this stage, the author insists on major changes, or on the withdrawal of the manuscript, he may be charged the printing costs.

10. Proofreading. Signs used in correcting proof and examples of their use will be found on the next 3 pages. Alterations in type which has been set are done by hand. Authors should call for only such changes as are essential. The insertion or deletion of a comma, for example, should be based on a compelling reason and not a whim.

If a number of consecutive words or symbols must be deleted or altered, they should be replaced if possible by material occupying 
nearly the same amount of space. If this is not done, the compositor will have to reset a number of neighboring lines to make the change. For example, if an author adds a twenty-letter phrase to the first line of a paragraph, the entire paragraph may have to be reset to make room for it. Such changes are costly and may lead to new errors.

It is important that, for each correction, two marks appear, one in the margin showing what the correction is, and one in the text showing where it is to go. The signs in the margins must have the order of the corresponding errors in the line, and they must be separated by a vertical bar $\mid$.

\section{TABLE II}

\section{SIGNS USED IN CORRECTING PROOFS}

N Delete; take out

¿ Close up

$\wedge$ Insert

\# Insert space

$\lceil$ Raise

L Lower

[ Move to left

] Move to right

Straighten type line at side of page

// Straighten lines

\$ Paragraph

center Put in middle of page or line

C Transpose

T/2 Transpose

D Turn inverted letter right side up
$X$ Change broken letter

Stet Let it stand as set

..... Let it stand as set

$\omega \cdot 6$. Wrong font, size or style

l.C. Lower case, not capitals

hom. Use Roman letter

bf. Use black type letters

$\odot$ Period

今 Comma

$\vee$ Apostrophe

2) Superior figure

仓 Inferior figure

$=/$ Hyphen

sC. Use small capitals

caps Use capitals

ital. Use italics 


\section{EXAMPLE SHOWING THE USE OF PROOF READING SIGNS}

of $B_{1}$ and $d_{1} d_{p}^{\prime}$ divides $d_{1} d_{p}^{\prime}+1$. Hence $\mathrm{B}$ is reducible to the foom $\uparrow /$ ital./ th (11.5) with diagonal terms $d_{1}, d_{1} d_{2}^{\prime}, \cdots, d_{1} d_{\rho}^{\prime}$ which proves (11.4).

12. Groups with@finite number of generators. We shall discuss certain properties of these groups culminating in the basic product decomosition (12.5).

(12.1) Definition. Let $B=\left\{g_{1}, \cdots, g_{n}\right\}, B^{\prime}=\left\{g_{1}^{\prime}, \cdots \cdot g_{n}^{\prime}\right\}$ be A.C. $/ \lambda$

\# two sets of elementspof $G$ containing the same number $n$ of elements. By

h a unimodulat transformation $\tau: B \rightarrow B^{\prime}$ is meant a system of elations $h=$

$$
r_{i}^{\prime}=\sum a_{i j} g_{j}, \quad\left\|a_{i j}\right\| \text { unimodular. }
$$

$\$$ The following proposition shows in how natural a manner unimodular transformations make their appearance in the theory of gro@ps with rom finite bases.

(12.3) Let $G$ be a group with a finite base $B=\left\{g_{1}, \cdots, g_{n \wedge}\right.$. In order $\}$ that $B^{\prime}=\left\{g_{1}^{\prime}, \ldots, g_{n}^{\vee}\right\}$ be a base for $G$ it is necessary and sufficient that $B^{\prime}$ be oblainable from $B$ by a unimodula $r$ transformation.

For any given set $B^{\prime}=\left\{g_{1}^{\prime}, \ldots, g_{n}^{\prime}\right\}$ of elements of $G$ there exist relations

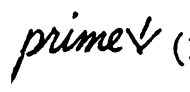

$$
g_{i}=\sum c_{i j} g_{j}, \quad C=\left\|c_{i j}\right\|_{\wedge}
$$

A necessary and sufficient condition in order that ${ }_{A}\left\{g_{i}^{\prime}\right\}$ be as base is $M / B^{\prime}=/ \mathcal{L}$

l.c. that the $\phi_{j}$ be expressible as lincar combinations of the $g_{i}^{\prime}$, or that there exist relations

$$
] g_{i}=\sum d_{i j} g_{i}^{\prime}, \quad D=\left\|d_{i}\right\| .[
$$

$[$ From this follows

$$
g_{i}=\sum d_{i j} c_{j k} g_{k j}
$$

- Hence since $B$ is a base we must have $\widehat{D C}=1$.)

This matrix relation yields $|D| \cap|C|=1$, and since the deter- hun un minants are integers we must have $|C|= \pm 1$. Thus in order that $B^{\prime}$ be a base $C$ must be unimodular, or the condition of (12.3) must be [ fulfilled. Conversely, if $j x$ is fulfilled, $C$ is unimodular and (12.5)

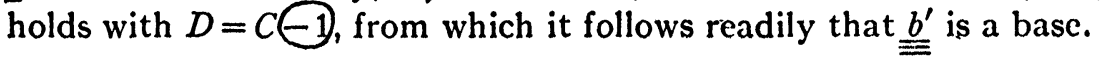




\section{THE PRECEDING PASSAGE PRINTED WITH ALL CORRECTIONS MADE}

of $B_{1}$ and $d_{1} d_{p}^{\prime}$ divides $d_{1} d_{p+1}^{\prime}$. Hence $B$ is reducible to the form (11.5) with diagonal terms $d_{1}, d_{1} d_{2}^{\prime}, \cdots, d_{1} d_{\rho}^{\prime}$ which proves (11.4).

12. Groups with a finite number of generators. We shall discuss certain properties of these groups culminating in the basic product decomposition (12.5).

(12.1) Definition. Let $B=\left\{g_{1}, \cdots, g_{n}\right\}, B^{\prime}=\left\{g_{1}^{\prime}, \cdots, g_{n}^{\prime}\right\}$ be two sets of elements of $G$ containing the same number $n$ of elements. $B y$ a unimodular transformation $\tau: B \rightarrow B^{\prime}$ is meant a system of relations

$$
g_{i}^{\prime}=\sum a_{i j} g_{j}, \quad\left\|a_{i j}\right\| \text { unimodular. }
$$

The following proposition shows in how natural a manner unimodular transformations make their appearance in the theory of groups with finite bases.

(12.3) Let $G$ be a group with a finile base $B=\left\{g_{1}, \cdots, g_{n}\right\}$. In order that $B^{\prime}=\left\{g_{1}^{\prime}, \cdots, g_{n}^{\prime}\right\}$ be a base for $G$ it is necessary and sufficient that $B^{\prime}$ be obtainable from $B$ by a unimodular transformation.

For any given set $B^{\prime}=\left\{g_{1}^{\prime}, \cdots, g_{n}^{\prime}\right\}$ of elements of $G$ there exist relations.

$$
g_{i}^{\prime}=\sum c_{i j} g_{j}, \quad C=\left\|c_{i j}\right\| .
$$

A necessary and sufficient condition that $B^{\prime}=\left\{g_{i}^{\prime}\right\}$ be a base is that the $g_{j}$ be expressible as lincar combinations of the $g_{i}^{\prime}$, or that there exist relations

$$
g_{i}=\sum d_{i j} g_{i}^{\prime}, \quad D=\left\|d_{i j}\right\| .
$$

From this follows

$$
g_{i}=\sum d_{i j} c_{j k} g_{k} \text {. }
$$

Hence since $B$ is a base we must have $D C=1$. This matrix relation yiclds $|D| \cdot|C|=1$, and since the determinants are integers $w c$ must have $|C|= \pm 1$. Thus in order that $B^{\prime}$ be a base $C$ must be unimodular, or the condition of (12.3) must be fulfilled. Conversely, if (12.3) is fulfilled, $C$ is unimodular and (12.5) holds with $D=C^{-1}$, from which it follows readily that $B^{\prime}$ is a base. 
TABLE III

\section{A LIST OF SIGNS AND SPECIAL CHARACTERS}

\begin{tabular}{|c|c|c|c|}
\hline 1. + & $* 36 . \cong$ & 71. । & 106. $\rightarrow$ \\
\hline 2. - & 37. $<$ & 72. || & 107. $\rightarrow$ \\
\hline 3. $x$ & 38. $>$ & *73. 引 & $*_{108 .} \rightleftarrows$ \\
\hline 4. $=$ & $*_{39}$ < & $* 74 . \quad$ & $*_{109 .} \rightleftarrows$ \\
\hline 5. $\div$ & $*_{40 .} \lessgtr$ & 75. 办 & $*_{110 .} \leftrightarrows$ \\
\hline 6. + & $*_{41}$. & 76. iㅔ & 111. $\sqrt{ }$ \\
\hline 7. - & 42. $\leq$ & $* 77 . \quad v$ & 112. $\sqrt[2]{ }$ \\
\hline 8. $X$ & 43. $\geq$ & 78. د & 113. $\sqrt[3]{ }$ \\
\hline 9. = & 44. $\leqq$ & 79. с & 114. $\sqrt[4]{ }$ \\
\hline 10. $\div$ & 45. $\geqq$ & $* 80 . \quad$ & 115. $\sqrt[3]{ }$ \\
\hline$*_{11}+$ & ${ }^{*} 46$. & 81. u & 116. $\sqrt[6]{ }$ \\
\hline 12. \pm & $*_{47}$. & 82. ก & 117. $\sqrt[7]{ }$ \\
\hline 13. \pm & 48. $\ngtr$ & *83. $\sum$ & 118. $\sqrt[8]{ }$ \\
\hline$*_{14} . \overline{7}$ & 49. $\Varangle$ & ${ }^{*} 84 . \bar{\Pi}$ & 119. $\stackrel{9}{ }$ \\
\hline$*_{15}$. 又 & 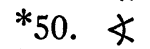 & 85. & 120. $\sqrt[n]{ }$ \\
\hline $16 . \doteq$ & $*_{51 . \quad \Varangle}$ & 86. $\rightarrow$ & 121. V \\
\hline 17. $\sim$ & 52. $\wedge$ & 87. $\leftarrow$ & 122. $\pi$ \\
\hline 18. $\sim$ & 53. V & 88. $\rightleftarrows$ & 123. $\nabla$ \\
\hline 19. $\approx$ & $*_{54 . ~ \gg}$ & 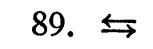 & 124. $\triangle$ \\
\hline 20. N & $*_{55} \ll$ & 90. ح & $*_{125}$. \\
\hline 21. $\simeq$ & 56. $\subset$ & 91. & 126. $\triangle$ \\
\hline 22. $\cong$ & 57. ᄀ & 92. $\leftrightarrow$ & 127. $\Delta$ \\
\hline 23. $\cong$ & 58. $\subseteq$ & 93. $\nwarrow$ & $*_{128 .} \angle$ \\
\hline 24. $\cong$ & 59. $\supseteq$ & 94. $\nearrow$ & $*_{129 . \quad / 8}$ \\
\hline 25. $x$ & 60. $\Varangle$ & 95. $\downarrow$ & 130. $\square$ \\
\hline 26. $\not$ & $* 61 . \quad \exists$ & 96. & 131. 曰 \\
\hline 27. $\neq$ & 62. $\in$ & 97. $\uparrow$ & 132. \\
\hline 28. $\neq$ & 63. $\cap$ & 98. $\bar{\imath}$ & 133. $\square$ \\
\hline 29. $=$ & 64. U & 99. ᄂ & 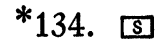 \\
\hline $30 . \equiv$ & 65. $\notin$ & 100. $\rightleftharpoons$ & 135. $\square$ \\
\hline 31. $\equiv$ & 66. U & 101. $\leftrightharpoons$ & $*_{136 .}$ \\
\hline 32. $\not \equiv$ & ${ }^{*} 67 . \Phi$ & 102. $\rightarrow$ & 137. $\diamond$ \\
\hline${ }^{*} 33 . \neq$ & *68. D & 103. $\uparrow$ & 138. \\
\hline $34 . \approx$ & 69. $\propto$ & 104. $\Rightarrow$ & 139. $\odot$ \\
\hline 35. & $70 . \infty$ & 105. $\Leftrightarrow$ & $*_{140 .}$ (S) \\
\hline
\end{tabular}

* An asterisk indicates symbols which are not available in a smaller font for use in inferior and superior position. 


\begin{tabular}{|c|c|c|c|c|c|}
\hline 141. ఠ & 162. & 183. & 1 & 204. & $b$ \\
\hline 142. @ & 163. & 184. & ' & 205. & $*$ \\
\hline 143. $\ominus$ & 164. & 185. & 1 & 206. & $*$ \\
\hline 144. $\theta$ & 165. & 186. & $"$ & 207. & $*$ \\
\hline 145. $\oplus$ & 166. & 187. & 1 & 208. & $m$ \\
\hline 146. $\otimes$ & 167. & 188. & $\circ$ & 209. & $\sigma^{x}$ \\
\hline 147. $\varnothing$ & 168. & 189. & : & 210. & 우 \\
\hline 148. ळ & 169. & 190. & . & 211. & $\overbrace{}^{\pi}$ \\
\hline 149. $\Upsilon$ & 170. & 191. & • & 212. & 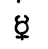 \\
\hline$*_{150 .}$ m & 171. & 192. & : & 213. & o \\
\hline 151. $\varnothing$ & 172. ........... & 193. & $\vdots$ & 214. & $\partial$ \\
\hline 152. & $173 . /$ & 194. & $\therefore$ & 215. & $\nabla$ \\
\hline 153. & 174.7 & 195. & $A$ & $*_{216 .}$ & $\Delta$ \\
\hline 154. ح & 175. $\perp$ & 196. & $\stackrel{s}{2}$ & $*_{217}$ & $\Delta$ \\
\hline$*_{155 .} \quad f$ & 176. $\vdash$ & 197. & $\star$ & 218 . & $*$ \\
\hline$*_{156 .} \quad f$ & 177. $上$ & 198. & $\star$ & 219. & $\dagger$ \\
\hline 157 & $*_{178 .}$ ヨ & 199. & * & 220. & $\ddagger$ \\
\hline 158. $\rangle$ & $*_{179}$ & 200. & $\#$ & 221. & $\S$ \\
\hline 159. / & 180. $\int$ & 201. & $\#$ & 222 . & I \\
\hline 160 & 181. $\mathcal{S}$ & 202. & ต & 223 & $\mathbb{1}$ \\
\hline 161. & 182. $\varnothing$ & 203. & $b$ & $*_{224 .}$ & $\ell$ \\
\hline
\end{tabular}

GREEK ALPHABET

\begin{tabular}{|c|c|c|c|c|c|}
\hline Alpha & $\mathrm{A} \alpha$ & Iota & I $\iota$ & Rho & $\mathbf{P} \rho$ \\
\hline Beta & B $\beta$ & Kappa & $\mathrm{K}_{\kappa}$ & Sigma & $\Sigma \sigma$ \\
\hline Gamma & $\Gamma \gamma$ & Lambda & $\Lambda \lambda$ & Tau & $\mathrm{T} \tau$ \\
\hline Delta & $\Delta \delta \partial$ & $\mathrm{Mu}$ & $\mathbf{M} \mu$ & Upsilon & $\Upsilon v$ \\
\hline Epsilon & $\mathbf{E} \epsilon$ & $\mathrm{Nu}$ & $\mathrm{N} \nu$ & Phi & $\Phi \phi \varphi$ \\
\hline Zeta & $\mathrm{Z} \zeta$ & $\mathrm{Xi}$ & 包 $\xi$ & Chi & $\mathrm{X} x$ \\
\hline Eta & $\mathrm{H} \eta$ & Omicron & 00 & Psi & $\Psi \psi$ \\
\hline Theta & $\Theta \theta \vartheta$ & $\mathrm{Pi}$ & $\Pi \pi$ & Omega & $\Omega \omega$ \\
\hline
\end{tabular}

